\title{
The Measurement of Musical Tone
}

\author{
WILLIAM H. STUBBINS
}

$\mathrm{P}$ RESENT METHODS and techniques for the measurement of sound permit the analysis of musical tone as acoustical phenomena. It is equally true that no one is certain at present that those aspects of the sound wave which are customarily measured are the aspects of primary importance in the auditory perception of musical tone. It is necessary that a correlation be made between these observations and the judgment of the ear. This can be accomplished by the cooperation of the scientist and the musician.

The first step in the direction of such cooperation lies in the understanding of the problem and in the willingness of each party to accept each other's techniques as supplementary to his own. There should be no desire to mechanize music nor to reject blindly the fact that sound is the basis of music. It is not unfair to the musician to say that the scientist already knows much more about sound than does the musician, but it is also true that the musician is much better equipped to employ sound in a particular and specialized manner than is the scientist. They both have much to offer each other through such cooperation; and the common gain, which will be the result of such combined effort, is a contribution to both science and to art.

Let us examine the present possibilities for the investigation of musical tone quality on the basis of what can be observed as physical phenomena of sound, in order to place the problem in a better perspective.
Suppose, for example, that it is desired to communicate information to another musician, in another part of the world, six months from now, concerning a clarinet tone which is to be played at this present time. This information is to be complete information, which will include all phases of what we may be able to say or to show about it. The simple technique of recording it with absolute fidelity and then merely playing it for the recipient of the information is not enough in this case. True, if the recording is as perfect as we can obtain, and the playback is also perfect, we will have transmitted the identical sound of the clarinet across the intervening interval of time. This accomplishment, however, is simply a timestretching elaboration of the same kind of communication which invokes the use of a qualitative vocabulary. We have been able to retain the clarinet tone of the present for future reference, but information which is needed for analysis and for better understanding is just as surely missing as if the recipient of the information had been present at the recording. Nothing has been lost, but nothing has been gained.

In addition to the perfect preservation of the presently played clarinet tone for future reference, it is also possible to provide other information concerning it by means of other techniques.

It is possible to present information in the form of a picture of the tone for purposes of visual analysis. It is 
possible to present information which will show visually and measure accurately the harmonic content, the amplitude and intensity, the frequency, the build-up and decay, and the comparative differences between all musical tones and this specific clarinet tone, or the similarities of this tone to others which have been played at any time and recorded for reference.

Whether this additional information will be of value to the musician or not will depend on the way in which it is used. The potentialities of communication are considered as self-evident.

\section{The Problem of Communication}

Communication is a basic characteristic of human beings. Man accomplishes communication by several means, by gesture, by pictorial indication, of which writing is a sub-script, and by sound, of which word noises and musical tones are primary categories. Of these means of communication, the matter of musical tone, its physical characteristics and its psychological implications, has never been adequately studied in order to realize the tremendous yield which is potential through proper use of the techniques of research available. ${ }^{1}$

A few of the results which may be anticipated from an adequate study of musical tone are better communication by means of this method of man's expression, better performance of the art of music, better and perhaps new instruments of music, better teaching of music, a more adequate terminology concerning the art of music, and a powerful technique for the study of the psychic aspects of the human being when the physical side of music and musical tone is better known. There

1H. F. Olson, Musical Engineering (New York: McGraw-Hill, 1952), p. vi. are undoubtedly valuable therapeutic possibilities which have only been touched upon so far by a few pioneers in this field. ${ }^{2}$

The basic physical property of music is sound-without which there can be no music. The production of sound for musical purposes is accomplished by the use of musical instruments, which are simply sound producers. The province of the study of such sound production, by such sound producers, for such purposes, is properly the science of musical acoustics. The human additives of the performer are limited by the physical considerations involved in this relationship. As the physical malformation of the cleft palate interferes with proper vocal production, so does the stuttering or stammering of the musical instrument interfere with proper and desirable musical tone production.

The savage with his reed flute cannot play Mozart's Concerto in D, not only because he knows nothing of Mozart, but also because his reed flute will not do it. We have here no quarrel with his expression as far as it goes, his simple melodies and his emotional satisfaction, but we cannot fail to acknowledge the vast potential of expressive possibilities implied by the development of civilization.

What the limitations of the various musical instruments may be, no matter what their present degree of refinement may be, should properly be determined by first attending to a study of the means used for musical sound production. The application of scientific techniques and methodology in the study of the physical characteristics of musical instruments is unde-

2Lewis, Burris-Meyer, and Cardinell, "Music As an Aid to Healing," Journal of the Acoustical Society of America, XIX (July 1947), 544. 546. 
niably an exploitation of the aforementioned potential of expression. ${ }^{3}$ Present knowledge concerning musical instruments is limited to the heritage of craft which has come down through the years with all of the mythology developed by tradition. By scientific determination, it should be possible to cut through this maze of contradiction and contra-distinction to basic principles of fact which would clarify and lead to a better understanding of the art of music in terms of reasonable, logical, and factual evidence concerning the tools of the musician, musical instruments.

\section{The Qualitative Vocabulary of Music}

The qualitative aspects of music have long been the subject of discussion among musicians. An extensive vocabulary for this purpose has been developed. ${ }^{4}$ But for the individual musician, the interpretive possibilities of musical expression have led to the formation of an individual subjectivism as concerns each and every musical term encountered, a fact which has provided him a communicative escape mechanism, based on the single standard of individual taste. ${ }^{5}$

Words are not music, and music is not of words, although the expressed qualitative esthetic experience may be parallel. ${ }^{6}$ If a musician would talk about music, he must use the words of the only vocabulary so far at his disposal, and he must reserve a place of retreat which is conveniently provided by his personal taste. That he

3H. Fletcher, "An Institute of Musical Science - A Suggestion," Journal of the Acoustical Society of America, XIX (July 1947), 527-531.

4R. W. Young, "Some Problems for Postwar Musical Acoustics," Journal of the Acoustical Society of America, XVI (October 1944), 105.

5D. H. Parker, Principles of Aesthetics (New

York: Silver Burdett Co., 1920), p. 176.

6Parker, pp. 185, 255. retreats so often is easily understandable; he might welcome a new vocabulary which would make his retreat less often necessary.

Now the human mind is a complex thing, and we know that the complexities of communication are fascinating as well as baffling. There is no reason to suppose that the qualitative vocabulary is going to be discarded; its boundaries are likely to increase with every musician who is born. However, description of the processes of music in terms of the already established vocabulary of the physical and biological sciences cannot fail to increase the probability of clearer thinking, more accurate analysis, and more efficient communication.

\section{The Quantitative Vocabulary}

The development of a quantitative vocabulary depends on the application of scientific techniques to the physical phenomena which are used in the art of music. The application of these techniques to the physical phenomena of music is no different from such applications in other fields of investigation. It is desired very simply to establish first of all a language of size. For this purpose, the problem may be designated as a problem in the measurement of the qualities of music, which qualities are those defined as the aspects of the phenomena of music which are measurable.

The terminology of music is usefully divided into three categories: first, terminology concerning the instrument; second, terminology concerning the player; and third, terminology concerning auditors other than the player.

\section{The Dimensions of Music}

The use of the physical phenomenon of sound for the purposes of music is 
conveniently considered as a dimensional relationship which provides us with specific operational limits.

Sound, as a natural phenomenon, is the result of vibration. It is defined as "an alteration in pressure, stress, particle displacement, particle velocity, etc., which is propagated in an elastic material, or the superposition of such propagated alterations." 7

We live surrounded by air. The motion of this air around us constitutes a central interest which is primary to everything that we do. This air moves about us in various ways, and when caused to move by the vibration of some elastic body, whether accidental or deliberate, results in the activation of our sensory equipment with particular reference to the ear, in such a way that the sense-perception of this air-motion causes us to experience what is known as the sensation of hearing. Psychologically, sound is for us a sensation produced by vibration. According to a second definition, "Sound is also an auditory sensation, usually evoked by the alterations described above." 8 This dichotomy of concept is of particular significance to the problem of musical analysis and immediately suggests the need for correlation if better understanding is to be achieved.

Three things are needed to accomplish the sound-cycle for us as human beings, a vibration producer or sound maker, a medium which can carry the vibrations produced by the vibration maker (in this case air), and a receiving instrument for the produced and carried vibrations, such as the human ear. ${ }^{9}$

Every material body which has the

7American Standard Acoustical Terminology: 1.040. Hereinafter abbreviated to ASAT.

8ASAT: 1.045 .

9W. Bartholomew, Acoustics of Music (New York: Prentice-Hall, Inc., 1942), p. 2. necessary properties for vibration has a time of vibration most natural to it depending on its size, its weight, and the stresses under which it may be vibrating. This time of vibration is called its natural period of vibration. It is the time necessary for the body to complete one back and forth movement or one vibration.

Vibration, or oscillation, is defined as "the variation, usually with time, of the magnitude of a quantity with respect to a specified reference when the magnitude is alternately greater and smaller than the reference." ${ }^{10} \mathrm{~A}$ periodic quantity is defined as "an oscillating quantity the values of which recur for equal increments of the independent variable," 11 and a period or primitive period of a periodic quantity is " the smallest value of the increment of the independent variable for which the function repeats itself." ${ }^{12}$ A cycle is defined as "the complete sequence of values of a periodic quantity which occur during a period," 13 and the frequency of the periodic quantity, "in which time is the independent variable, is the number of periods occuring in unit time."14 The number of cycles which a body may complete in one second is designated as its fundamental frequency, "the frequency ... which has the same period as the periodic quantity." 15

Bodies with short periods thus vibrate more frequently in the same time than do bodies with longer periods. Their frequency of vibration is said therefore to be greater. In order to produce audible sound, or the sensation of hearing, vibrating bodies must have some frequency between approxi-

\footnotetext{
10ASAT: 1.040 .

11ASAT: 1.045 .

12ASAT: 1.050 .

13ASAT: 1.055 .

14ASAT: 1.060 .

15ASAT: 1.095 .
} 
mately twenty and twenty thousand cycles per second. This is the natural limitation of the human ear and consequently the sound limit of the frequencies used in music.

In order to be used as sound in music, the frequency of vibration, within these limits, must be produced in such a manner that it is continuous and steady for a long enough time interval to be used as a point of reference. This steady-state of vibration at a certain frequency allows the sound to be described as having a certain pitch. Pitch is defined as "that attribute of auditory sensation in terms of which sounds may be ordered on a scale extending from low to high, such as a musical scale."16 The musician refers to the steady-state frequency of a tone primarily as its pitch.

The wideness of range of movement made by any body during its period of vibration constitutes its amplitude of vibration. The result of amplitude is what the musician refers to as the loudness of the pitch. Amplitude is defined as "the largest value which the quantity attains." ${ }^{17}$ Loudness is defined as "the intensive attribute of an auditory sensation, in terms of which sounds may be ordered on a scale extending from soft to loud." 18

The duration of a steady-state of a certain frequency permits the use of the sound for longer or shorter intervals of time if the sound producer is properly controlled. This permits the use of the sound for musical purposes for intervals of greater or less duration. Thus, individual sounds may be given what musicians call a rhythmic relationship.

On the basis of this brief analysis of the properties of sound and its use

16ASAT: 5.005 .

17ASAT: 1.070 .

18ASAT: 5.015 . in music, a plot of three dimensions of music may be constructed, as in Figure 1. There is no note which may be played or sung as used in music which does not fall in this three-dimensional plot.

\section{Tone Quality}

While the three-dimensional plot of sound as used in music is conveniently limited as shown in Figure 1, it is obvious to any musician and to any scientist interested in these matters that a very important aspect of sound and of music is missing. This missing property is what is known generally as tone quality.

Timbre or tone quality is defined as "that attribute of auditory sensation in terms of which a listener can judge that two sounds similarly presented and having the same loudness and pitch are dissimilar." ${ }^{19}$ It is the characteristic which enables a musician to judge that he is hearing a clarinet for example, instead of a violin, or an oboe instead of a French horn, even though they may be playing the same pitch at the same dynamic level.

Any body used as a sound producing medium for musical purposes vibrates in a number of secondary motions in addition to the basic motion of its natural period. These secondary motions also produce vibrations, and hence sounds, although usually fainter than the principal vibration. The basic pitch produced by the basic motion is called the fundamental, and the secondary sounds are in music called harmonics, overtones, or partials.

The fundamental tone is defined as "the component in a periodic wave corresponding to the fundamental frequency, or the component tone of lowest pitch in a complex tone." ${ }^{20} \mathrm{~A}$ tone is defined as "a sound wave capable

19ASAT: 6.050 .

20ASAT: 1.095 . 


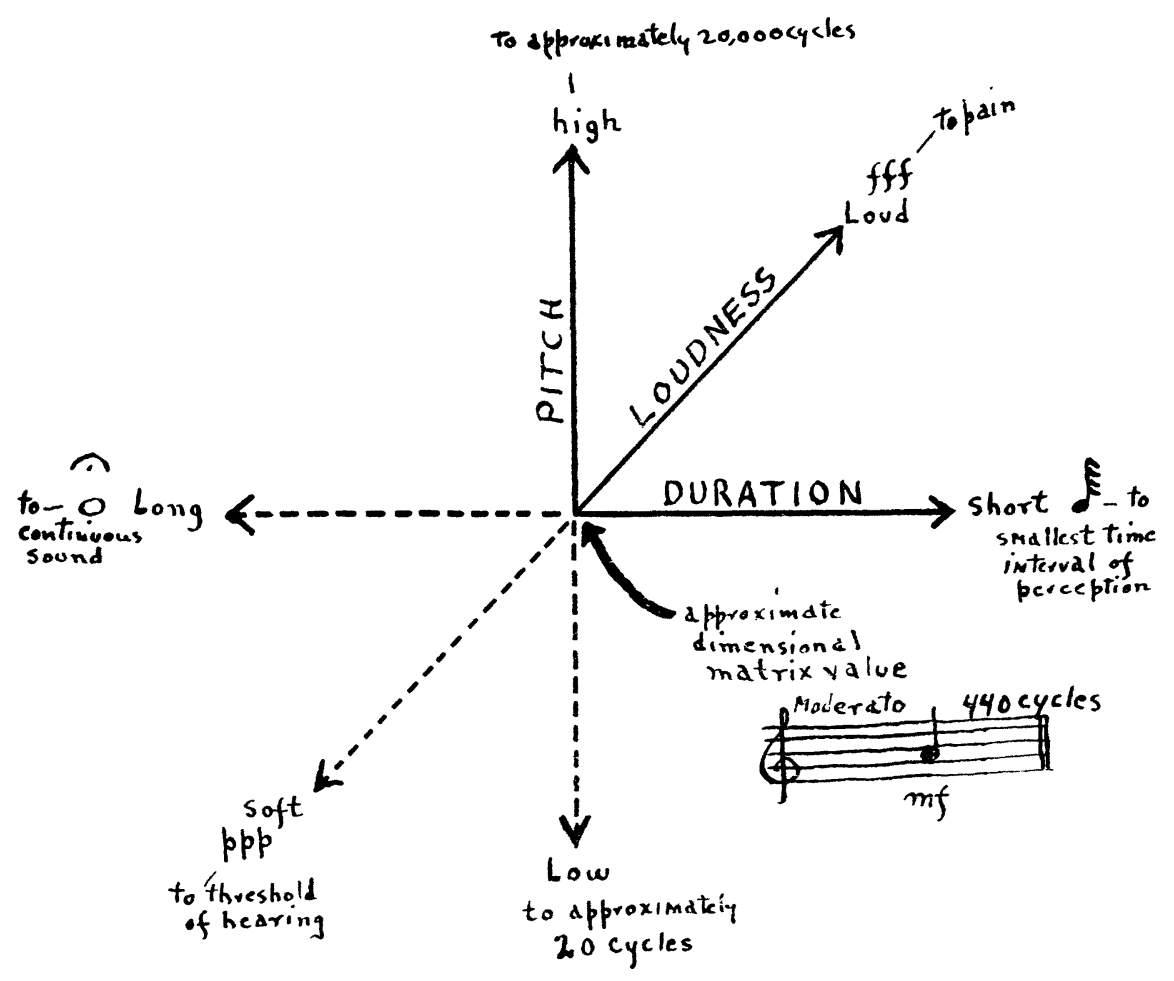

Figure 1

of exciting an auditory sensation having pitch, or a sound sensation having pitch." ${ }^{21}$ A simple tone is defined as "a sound wave, the instantaneous sound pressure of which is a simple sinusoidal function of the time, or a sound sensation characterized by its singleness of pitch." ${ }^{22}$ A complex tone is defined as "a sound wave produced by the combination of simple sinusoidal components of different frequencies, or a sound sensation characterized by more than one pitch." ${ }^{23}$

An overtone is defined as "a physical component of a complex sound having a frequency higher than that of the basic frequency, or a component of a complex tone having a pitch higher than that of the fundamental pitch." 24 A partial is defined as "a physical component of a complex tone, or a component of a sound sensation which may be distinguished as a simple tone that cannot be further analyzed by the ear and which contributes to the character of the sound." ${ }^{25}$ A harmonic is defined as "a partial whose frequency is an integral multiple of the fundamental frequency." ${ }^{26}$ It is inaccurate to use these terms as synonyms, which is a common weakness of the qualitative vocabulary.

The sum total of the various motions in a vibrating body with respect to their strength and to the possibilities of their combination is apparently 
what the human ear uses as its basis for the judgment of what the musician calls tone quality. It is apparent that in addition to our simple three-dimensional limit of sound as used in music and shown in Figure 1, that we must reckon with another property of musical sound which is present under all conditions for the production of music.

As an example of the quantitative measurement of sound as used in music, the matter of tone quality is of prime importance because it represents an area of great interest of which at present not too much is known. The qualitative vocabulary of music has in some measure been able to function with respect to the threedimensional limits of sound as described in the discussion of pitch, dynamics, and rhythm, but in the case of tone quality the breakdown is obvious.

Generally speaking, musical ears do not disagree too violently with respect to matters of pitch, or intonation, as it is commonly called. Neither is there any particular difficulty in reaching agreement concerning rhythmical subdivisions or dynamic variations. But as to tone quality, its nature, its description, its differences, and its effect, musicians all but become inarticulate, and at the very best, unintelligible. Here is the point of greatest need for a quantitative vocabulary, and here is the opportunity for the most significant application of scientific techniques.

The term tone quality is used by musicians for the purposes of describing first, the tone production of the musical instrument, its nature or function; second, methods and mannerisms of individual performance, the control of the instrument by the player, or the function of the player; and third, certain conceptual and emotional values which may be the effect of musical expression.

Tone quality in the first sense, as applied to what the instrument produces, may be referred to by such descriptive terms as dull, heavy, dark, bright, light, brilliant, etc.

When tone quality is referred to in the second sense, in terms of the sound as controlled by the player due to certain physical activities on his part, the same descriptive words may again be employed but modified by personal reference as in the phrases, "so and so's tone is" or "so and so's sound is."

Or again, if tone quality is referred to in the third sense, as concerns a description of the effect of musical expression, the same words may again be used, but modified in such a way as to express an imaginative concept such as "the transparent, light sound of the strings," or the "stinging brilliance of the trumpets," or "the dark sonorities of tonal architecture."

Thus, if a musician is confronted with the blunt term, tone quality, he may think of his instrument in terms of its mechanical function, or of the sound which he produces when playing it, or of the subtle differences which he imagines may be achieved when the instrument is being played by him for the purpose of producing some special effect of style in the expression of music.

The interrelations of these categories of definition are illustrated by Figure 2. The arrows represent the several uses of the same descriptive terms applied under different modes of usage. Tone quality in the first usage, that having to do with what the instrument may produce as its function (that is, what it is designed for, with the implied limitation of this efficiency), is a consideration of the player with re- 


\section{(music)}

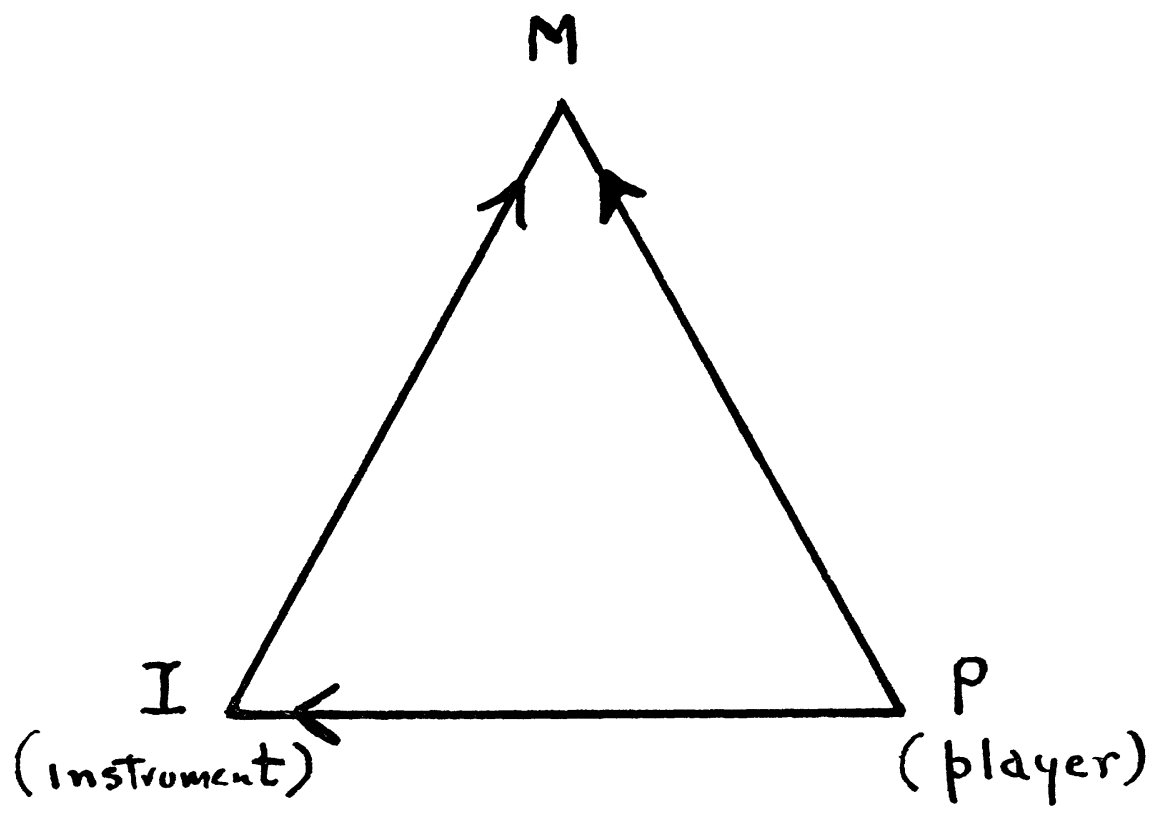

FIGURE 2

spect to the instrument as a purely mechanical construction. This may be represented symbolically as $\mathrm{P} \rightarrow \mathrm{I}$.

This mechanical function of the instrument in terms of its own efficiency has a potential capacity as concerns the production of sound for the purposes of music, which in this case is its total value as a mechanical construction, I $\rightarrow$ M.

The mechanical function of the instrument as controlled by the player for the expression of music is a sum total of the mechanical efficiency of the instrument and the physical control of the player, $\mathrm{P} \rightarrow \mathrm{I} \rightarrow \mathrm{M}$.

The sound used for the expression of music as an art, although a product of this psychoacoustical activity, is regarded as an independent phenomenon when the considerations of the player are directed toward it in terms of musical expression, $\mathrm{P} \rightarrow \mathrm{M}$.
If a substitution for the player, $P$, in Figure 2 is made by an audience, $A$, as in Figure 3, the effect of the mechanical efficiency of the instrument and the physical activity of the player is that of a sum total of PI, and PI becomes a complex factor which may be written symbolically as (PI). The effect of the music in this case is $\mathrm{M} \rightarrow \mathrm{A}$, and the total effect of the complex situation may be expressed as $[(\mathrm{PI}) \rightarrow \mathrm{M}]+[\mathrm{M} \rightarrow \mathrm{A}]+$ $[(\mathrm{PI}) \rightarrow \mathrm{A}]$ equals $[(\mathrm{PI}) \mathrm{M}]+$ [(PI)A] equals $[(\mathrm{PI}) \mathrm{M} \rightarrow \mathrm{A}]$.

Tone quality may therefore be described as in Figure 2 by the player in terms of $\mathrm{I},[\mathrm{P} \rightarrow \mathrm{I}]$, or $[\mathrm{P} \rightarrow \mathrm{I} \rightarrow \mathrm{M}]$; and in Figure 3 by the audience as characteristic of the instrument alone, or due to any of the following relationships: $[\mathrm{A} \rightarrow \mathrm{I}]$, $[\mathrm{A} \rightarrow \mathrm{P}],[\mathrm{A} \rightarrow(\mathrm{PI})],[(\mathrm{PI}) \rightarrow \mathrm{M}]$, or $[\mathrm{M} \rightarrow \mathrm{A}]$. 


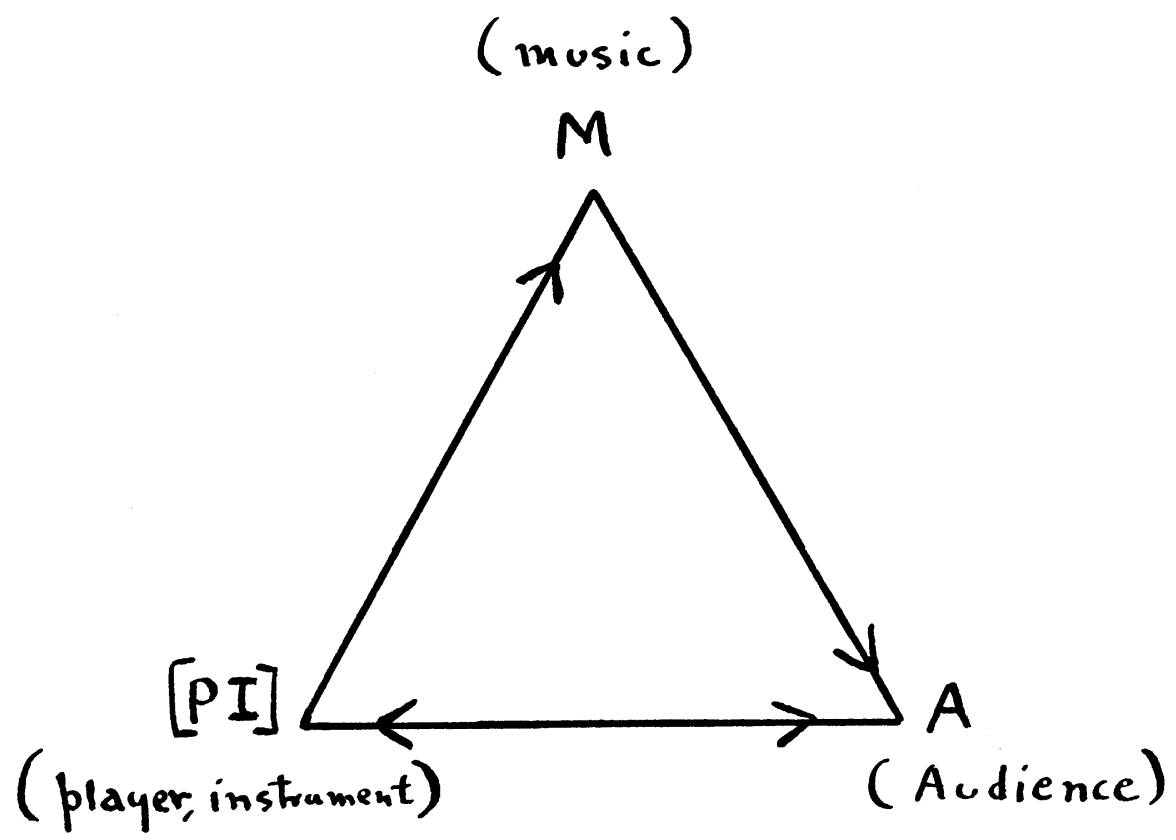

Figure 3

This dilemma of understanding among musicians according to their use of the descriptive possibilities of the term tone quality emphasizes the need for quantitative analysis.

\section{Classification of Musical Instruments}

There are two general classifications of musical instruments which are immediately recognizable. There are those instruments in which the tone production is greatly dependent on the player and there are those instruments in which the tone production is more or less a mechanical function of the instrument itself, this function being controlled by the player. Obviously, this classification of instruments is mechanical, but it provides us with the means of separating the functions of the player and the instrument for further analysis.

In attempting to describe the tone quality of instruments of the first category, we are much more concerned with what the player does than we are when describing the tone quality of instruments of the second category. With regard to the description of intonation, we are exclusively concerned with what the player does with instruments of the first category, and not at all concerned with what he does with instruments of the second category. A pre-set instrument of the second category leaves the player with no control over this matter whatsoever, while in the former case, intonation presents one of the greatest difficulties of performance.

Most of the violent disagreements concerning the definition of tone quality spring from this difference in musical instruments. To the degree that the player has control of the tone generation of his instrument, and to the degree of flexibility of adjustment which the instrument possesses, the more difficult it becomes to define and measure musical instrument tone qual- 
ity. Discussions of this nature are too often referred to as simply psychoacoustical problems. While it is true that they are, there is considerably more than psychoacoustical values involved, and the correlation of a quantitative analysis is to be desired.

\section{The Judgment of Tone Quality}

In addition to the description of musical instrument tone quality by the use of terms such as dull, heavy, dark, light, bright, brilliant, etc. (as applied to the three references: the instrument, the player, and the music), the musician quite simply uses as a basis for first judgment on hearing a musical tone, the terms good or bad. This first quick and automatic judgment is based on acceptance or rejection of the tone as being worthy of further description. Good tones merit further discussion and classification according to the extent of the qualitative vocabulary, while bad tones need improvement before their subleties are discussed.

If some basis of this first judgment of the musician can be established to the effect that there is a measurable difference between tones qualitatively referred to as good and bad, and if this quantitative difference can then be correlated with and substantiated by the musician's ear, we will have at least limited our scope of reference and can proceed further.

\section{Measurement of Tone Quality}

In the first analysis of physical data concerning musical tone it is found that in order for a sound to be defined as a musical tone that it must have a fixed, or measurable frequency which has a duration sufficient for reference and which has a definite, or measurable intensity. The frequency, the duration, and the intensity can be accurately measured by scientific in- struments and can less accurately be determined by the ear.

It is simple enough to collect physical data concerning musical tone, but it is not a simple matter to determine what these data may mean in terms of the ear. The scientific instrument for measurement is analytic, the ear is synthetic. The ear subtracts, adds, selects that which it wishes to hear -it even may add certain components of its own to the sound which is to be described, but it hears as a whole. The scientific instrument is impartial; the ear is prejudiced by all of the concomitant factors of the complex associations of thinking which make up the nature of the human being.

No instrument of sound measurement can be substituted for the human ear, nor can the human ear ever analyze sound with the impartial reference of a sound measurement device. Certain differences of sound for the ear are, however, the same differences for the measuring device, and the reference to these differences constitutes our quantitative vocabulary.

If the sound produced by a musical instrument does not possess the properties which satisfy its definition as a musical tone with respect to the measurement of the scientific instrument nor to the 'hearing' of the ear, there are but two alternatives which may be in effect: the musical instrument is at fault mechanically, or the player is not exercising the degree of control possible and necessary for the proper mechanical functioning of the instrument.

\section{Conclusion}

Since by definition a musical instrument is a sound producer designed to produce musical tones, the nature of the instrument plus the efficiency of the mechanical function of the instrument plus the efficiency of the player's 
control (which is a skill quotient) should also be said that the materials gives musical tones of a particular of art, the colors of the painter, the quality. Introduced variables of de- words of the poet, and the musical sign, mechanical function, and play- tones of the musician are dependent on er's control all produce differences in physical phenomena without which art tone quality.

The nature of the instrument, the mechanical function of the instrument, as well as the player's control, all may be measured by instruments and be heard by the ear, and introduced variables may also be measured and may be heard.

It can be argued that art is self sufficient and complete in itself. It University of Michigan could not be. Better and more complete understanding of these materials and their uses cannot fail to increase the efficiency of the artistic process and might perhaps give rise to a broader scope of artistic development simply on the basis of the added freedom of the mind which comes about through greater understanding. 